\title{
Über Bewegungsinvarianten und elementare Geometrie in einer Minimalebene.
}

Von Ludwig Berwald in Grïnwald bei München.

In einer schon vor längerer Zeit erschienenen Abhandlung ${ }^{1}$ ) hat Herr Study für die Geometrie in einer Euklidischen Ebene das Fundamentalproblem der Theorie der Bewegungsinvarianten gelöst: alle ganzen irreduziblen Bewegungsinvarianten in einem unbegrenzten System von Punkten und Geraden sowie die zwischen diesen Invarianten stattfindenden irreduziblen Relationen aufzustellen.

Die vorliegende Arbeit soll nun vor allem zeigen, daß das von Herrn Study eingeschlagene Verfahren ohne besondere Schwierigkeit auch zur Lösung der (wesentlich einfacheren) Aufgabe tauglich ist, alle ganzen irreduziblen Invarianten gegenüber den automorphen Ähnlichkeitstransformationen einer Minimalebene in einem unbegrenzten System von Punkten und Geraden dieser Ebene aufzustellen, und die $z$ wischen diesen Invarianten stattfindenden irreduziblen Relationen erschöpfend anzuge eben.

Trotz ihrer prinzipiellen Einfachheit hat die Lösung dieser Aufgabe (die einer noch etwas allgemeineren Formulierung fähig ist ${ }^{2}$ ) ein erhebliches geometrisches Interesse. Denn erst, wenn alle ganzen irreduziblen Ähnlichkeitsinvarianten der Minimalebene bestimmt sind, lassen sich in systematischer Weise für die einfachsten Figuren von Punkten und Geraden der Minimalebene solehe homogene Funktionen $0^{\text {ten }}$ Grades der Koordinaten ihrer Elemente bilden, welche bei den Transformationen gewisser Untergruppen der automorphen Ähnlichkeiten ungeändert bleiben, also den Charakter "absoluter Invarianten" besitzen.

Unter den erwähnten Untergruppen, die Herr Beck neuerdings, ${ }^{3}$ ) zum Teil sehr eingehend, studiert hat, sind für die ana-

1) E. Study, Über Bewegungsinvarianten und elementare Geometrie I. Ber. Ges. Ipz. (math.-phys.) 48 (1896), S. 649-664 (weiterhin als „BJ." zitiert).

2) Vgl. Nr. 1 ff. des folgenden.

3) H. Beck, Zur Geometrie in der Minimalebene, Sitz.-Ber. Berl. Math. Ges. $12(1912 / 13)$, S. 14-30 (weiterhin als "ME." zitiert). - Diese Abhandlung, die sich größtenteils mit dem Studium der automorphen Bewegungen einer Minimalebene beschäftigt, enthält naturgemäb auch bereits einen Teil der im folgendem abzuleitenden Resultate; doch ist der Standpunkt, von dem aus sie gewonnen werden, ein von dem unsrigen verschiedener. - 
lytische Geometrie in der Minimalebene von besonderer Wichtigkeit die Bewegungen derGrenzgruppe, eine dreigliedrige invariante Untergruppe der antomorphen Ähnlichkeiten der Ninimalebene, die aus den automorphen Bewegungen einer Euklidisch en Ebene hervorgeht, wenn man mit dieser (in geeigneter Weise) den Grenzübergang zur Minimalebene rornimmt. ') Die Bew egu u gen der Grenzgruppe sind nämlich die allgemeinste Untergruppe der automorphen Ähnlichkeiten einer Ninimalebene, für welche die Figur zweier (eigentlicher) Punkte, diejenige zweier (Euklidischer) Geraden, und die Figur eines (eigentlichen) Punktes und einer (Euklidischen) Geraden sämtli $\mathrm{ch}$ je eine absolute Invariante besitzen: im Gegensatz zur Euklidischen Ebene, wo diese Eigenschaft allen automorphen Bewegungen zukommt. Eine .Elementargeometrie", die in ihrem Aufbau derjenigen der Euklidischen Ebene entspricht, läßt sich somit in der Minimalebene nur dann begründen, wenn man ihr als Fundamentalgruppe die Bew egungen der Grenzgruppe zu Grande legt.

Es zeigt sich dann weiter, dal in dieser "Elementargeometrie in der Minimalebene" metrische Dualitä "berrscht: die bloße Vertauschung von Punkt- und Geradenkoordinaten füht - bis auf einen Zahlenfaktor - jede absolute Invariante von Punkten (und Geraden) in eine solche der entsprechenden Geraden (und Punkte) über, und umgekehrt. Diese Eigenschaft der Geometrie der Bewegungen der Grenzgruppe, auf der ein großer Teil ihrer Einfachheit und Durchsichtigkeit beruht, scheint noch nicht bemerkt worden $\mathrm{zu}$ sein.

Über das Dargelegte hinaus beschränkt sich die vorliegende Arbeit durchaus auf die Ableitung solcher geometrischer Sätze, die unmittelbar aus den Definitionen der absoluten Invarianten zweier und dreier Punkte oder Geraden sowie eines Punktes und einer Geraden folgen. Insbesondere werden eine Reihe von Punlttund Geradenörtern in der Minimalebene angegeben und diejenigen Relationen zwischen den absoluten Invarianten der Ecken und Seiten eines Dreieckes aufgestellt, wclche die Metrik dieser Figur beherrschen.

1. Wir betrachten in einem, reellen oder komplexen, ternären Gebiete, ${ }^{2}$ ) geometrisch gesprochen in einer Ebene, diejenigen (nicht-

Der exste, der sich mit den automorphen Bewegungen der Minimal e ben e beschäftigt hat, ist unseres Wissens Herr Study gewesen (Zur Differentialgeometrie der analytischen Curven, Trans. Amer. Math. Soc. 10 (1909), S. $1-50)$.

1) H. Beck, ME., S. 24 ff., wo dieser Grenzïbergang ausgekührt wird. Auch der Name "Grenzgruppe" stammt von Herrn B e ck.

2) Wir nennen ein ternäres Gebiet reell oder komplex, je nachdem die Koordinaten $\left(x_{1}: x_{2}: x_{3}\right)$ der Punkte $x$ und $\left(u_{1}: u_{2}: u_{3}\right)$ der Geraden $u$ des Gebietes lediglich dem Bereiche der reellen Zahlen, oder auch demjenigen der (gewöhnlichen) komplexen Zahlen entnommen werden dürfen. Unter einer $k \cdot g$ li ed rig e $n$ Gruppe von Transformationen eines komplexen Gebietes verstehen wir dann eine solche Transformationsgruppe, die von $k$ komplexen (also $2 k$ reellen) Parametern abbängt. 
entarteten) Kollineationen, welche eine Gerade $l$ und einen mit ihr vereinigt liegenden Punkt $\lambda$

$$
\left.(l \lambda)=0^{1}\right)
$$

in Ruhe lassen. Diese Kollineationen bilden eine fünfgliedrige Untergruppe $K_{5}$ der achtgliedrigen Gruppe $K_{8}$ der automorphen Kollineationen der Ebene.

Unter einer ganzen Invariante einer Gruppe $K$ von automorphen Kollineationen der Ebene verstehen wir ${ }^{2}$ ) eine solche allseitig-homogene Funktion der als unabhängig, veränderlich gedachten Koeffizienten irgend welcher algebraiseher Formen $f_{i}\left(x_{1}, x_{2}, x_{3} ; u_{1}, u_{2}, u_{9}\right)$, die bei Ausführung einer Kollineation der Gruppe $K$ auf die Punkte $x$ und die Linien $u$ der Ebene sich mit einem nur von den Transformationskoeffizienten abhängigen Faktor reproduziert.

Unsere nächste Aufgabe ist der Aufbau der ganzen Invarianten der Gruppe $K_{5}$, von beliebig vielen Punkten $x, y, z$ und Geraden $u, v, w \ldots$ der Ebene, aus ihren einfachsten Elementen. Als solche erweisen sich die folgenden simultanen Invarianten der allgemeinen projektiven Gruppe:

$$
\left.\begin{array}{lll}
(u v w), & (u x), & (x y z), \\
(l u v), & (l x), & \\
& (u \lambda), & (x y \lambda),
\end{array}\right\}
$$

samt den aus ihnen durch beliebige Vertauschungen der Punkte $x, y, z \ldots$ untereinander und der Geraden $u, v, v \ldots$ untereinander hervorgehenden neuen Invarianten. Es gilt nämlich der Satz:

Jede ganze rationale Funktion der "Typen" (2), die homogen ist in bezug auf alle Symbole $x, y \ldots ; u, v \ldots$ (d. h. in bezug auf jedes einzelne der Systeme von drei Größen $\left.x_{i}, y_{i} \ldots ; u_{i}, v_{i} \ldots\right)$, aber nicht notwendig homogen in bezug auf die Symbole $l$ und $\lambda$, ist eine ganze Invariante der Gruppe $K_{5}$, und umgekehrt.

Enthält eine solche ganze Invariante nur die Typen der ersten bezw. der ersten und zweiten (ersten und dritten) Zeile von (2), so ist sie bereits ganze Invariante der Gruppe $K_{8}$ bezw. derjenigen sechsgliedrigen Untergruppe $K_{6}\left(K_{6}^{*}\right)$ von $K_{8}$, welche die Gerade $l$ (den Punkt $\lambda$ ) in Ruhe läßt.

1) Wir benutzen für die aus den Koordinaten dreier Geraden oder Punkte gebildeten Determinanten und fïr die linearen Formen die folgenden Symbole:

$$
\begin{gathered}
(u v w)=\left|u_{1} v_{2} w_{3}\right|, \quad(x y z)=\left|x_{1} \iota_{2} z_{3}\right|, \\
(u x)=u_{1} x_{1}+u_{2} x_{2}+u_{3} x_{3} .
\end{gathered}
$$

2) Diese Definition folgt (nahezu wörtlich) der von Herrn Study für die ganzen Bewegungsinvarianten aufgestellten (BJ., S. 650). Auch das weitere in dieser Nummer befolgte Verfahren ist dem seinigen nachgebildet (vgl. BJ., S. $651 \mathrm{ff}$.). 
2. Die Invariantentypen (2) sind nun durch gewisse irreduzible Id entität en miteinander verknüpft, die sich ersch öpfend angeben lassen. Es sind die folgenden:

a) 1. $\left(u_{2} u_{3} u_{4}\right)\left(u_{1} x\right)-\left(u_{3} u_{4} u_{1}\right)\left(u_{2} x\right)+$

$$
+\left(u_{4} u_{1} u_{2}\right)\left(u_{3} x\right)-\left(u_{1} u_{2} u_{3}\right)\left(u_{4} x\right)=0
$$

2. $\left(u_{2} u_{3} u_{4}\right)\left(u_{1} v w\right)-\left(u_{3} u_{4} u_{1}\right)\left(u_{2} v w\right)+$

$$
+\left(u_{4} u_{1} u_{2}\right)\left(u_{3} v w\right)-\left(u_{1} u_{2} u_{3}\right)\left(u_{4} v w\right)=0
$$

b) 1. $\left(x_{2} x_{3} x_{4}\right)\left(u x_{1}\right)-\left(x_{3} x_{4} x_{1}\right)\left(u x_{2}\right)+-$

2. $\left(x_{2} x_{3} x_{4}\right)\left(x_{1} y z\right)-\left(x_{3} x_{4} x_{1}\right)\left(x_{2} y z\right)+$

$$
+\left(x_{4} x_{1} x_{2}\right)\left(u x_{3}\right)-\left(x_{1} x_{2} x_{3}\right)\left(u x_{4}\right)=0 \text {, }
$$

c)

$$
\begin{gathered}
+\left(x_{4} x_{1} x_{2}\right)\left(x_{3} y z\right)-\left(x_{1} x_{2} x_{3}\right)\left(x_{4} y z\right)=0, \\
(u v w)(x y z)-|(u x)(v y)(w z)|=0 .
\end{gathered}
$$

a) 1. $\left(l u_{2} u_{3}\right)\left(u_{1} x\right)+\left(l u_{3} u_{1}\right)\left(u_{2} x\right)+$

$$
+\left(l \quad u_{1} u_{2}\right)\left(u_{3} x\right)-\left(u_{1} u_{2} u_{3}\right)(l x)=0,
$$

2. $\left(l \quad u_{2} u_{3}\right)\left(u_{1} v w\right)+\left(l \quad u_{3} u_{1}\right)\left(u_{2} v w\right)+$

$$
+\left(l \cdot u_{1} u_{2}\right)\left(u_{3} v w\right)-\left(u_{1} u_{2} u_{3}\right)(l v w)=0,
$$

b) $\quad\left(u_{2} u_{3} u_{4}\right)\left(l u_{1} v\right)-\left(u_{3} u_{4} u_{1}\right)\left(l u_{2} v\right)+$

$$
+\left(u_{4} u_{1} u_{2}\right)\left(l u_{3} v\right)-\left(u_{1} u_{2} u_{3}\right)\left(l u_{4} v\right)=0
$$

c) $\quad\left(x_{2} x_{3} x_{4}\right)\left(l x_{1}\right)-\left(x_{3} x_{4} x_{1}\right)\left(l x_{2}\right)+$ $+\left(x_{4} x_{1} x_{2}\right)\left(l x_{3}\right)-\left(x_{1} x_{2} x_{3}\right)\left(l x_{4}\right)=0$,

d)

$$
(l u v)(x y z)-\left|\begin{array}{lll}
(l x) & (u x) & (v x) \\
(l y) & (u y) & (v y) \\
(l z) & (u z) & (v \hat{z})
\end{array}\right|=0,
$$

e) $\quad\left(l u_{1} u_{2}\right)\left(l u_{3} u_{4}\right)+\left(l u_{1} u_{3}\right)\left(l u_{4} u_{2}\right)+\left(l u_{1} u_{4}\right)\left(l u_{2} u_{3}\right)=0$.

a) 1. $\left(x_{2} x_{3} \lambda\right)\left(u x_{1}\right)+\left(x_{3} x_{1} \lambda\right)\left(u x_{2}\right)+$

$$
+\left(x_{1} x_{2} \lambda\right)\left(u x_{3}\right)-\left(x_{1} x_{2} x_{3}\right)(u \lambda)=0
$$

2. $\left(x_{2} x_{3} \lambda\right)\left(y z x_{1}\right)+\left(x_{3} x_{1} \lambda\right)\left(y z x_{2}\right)+$

$$
+\left(x_{1} x_{2} \lambda\right)\left(y z x_{3}\right)-\left(x_{1} x_{2} x_{3}\right)(y z \lambda)=0
$$

b) $\quad\left(x_{2} x_{3} x_{4}\right)\left(x_{1} y \lambda\right)-\left(x_{3} x_{4} x_{1}\right)\left(x_{2} y \lambda\right)+$

$$
+\left(x_{4} x_{1} x_{2}\right)\left(x_{3} y \lambda\right)-\left(x_{1} x_{2} x_{3}\right)\left(x_{4} y \lambda\right)=0,
$$

c) $\quad\left(u_{2} u_{3} u_{4}\right)\left(u_{1} \lambda\right)-\left(u_{3} u_{4} u_{1}\right)\left(u_{2} \lambda\right)+$

$$
+\left(u_{4} u_{1} u_{2}\right)\left(u_{3} \lambda\right)-\left(u_{1} u_{2} u_{3}\right)\left(u_{4} \lambda\right)=0 \text {, }
$$

d)

$$
(x y \lambda)(u v w)-\left|\begin{array}{lll}
(u x) & (v x) & (w x) \\
(u y) & (v y) & (w y) \\
(u \lambda) & (v \lambda) & (w \lambda)
\end{array}\right|=0,
$$

e) $\quad\left(x_{1} x_{2} \lambda\right)\left(x_{3} x_{4} \lambda\right)+\left(x_{1} x_{3} \lambda\right)\left(x_{4} x_{2} \lambda\right)+\left(x_{1} x_{4} \lambda\right)\left(x_{2} x_{3} \lambda\right)=0$. 


$$
\left.\begin{array}{l}
\text { a) } \quad\left(l u_{2} u_{3}\right)\left(u_{1} \lambda\right)+\left(l u_{3} u_{1}\right)\left(u_{2} \lambda\right)+\left(l u_{1} u_{2}\right)\left(u_{3} \lambda\right)=0, \\
\text { b) } \quad\left(x_{2} x_{3} \lambda\right)\left(l x_{1}\right)+\left(x_{3} x_{1} \lambda\right)\left(l x_{2}\right)+\left(x_{1} x_{2} \lambda\right)\left(l x_{3}\right)=0, \\
\text { c) } \left.\quad(l u v)(x y \lambda)-\left|\begin{array}{ccc}
(l x) & (u x) & (v x) \\
(l y) & (u y) & (v y) \\
0 & (u \lambda) & (v \lambda)
\end{array}\right|=0 .{ }^{1}\right)
\end{array}\right\}
$$

In dieser Zusammenstellung haben wir mit 1. und 2. immer zwei solche Relationen unterschieden, von denen die zweite aus der ersten durch eine Substitution des Typus:

hervorgeht.

$$
u=\widehat{x y} \quad \text { oder } \quad x=\widehat{u v}
$$

Die Struktur des Systems der Relationen (3)-(6) gibt zu einigen Bemerkungen Anlaß:

Die Relationen (3) verbinden nur Invarianten der allgemeinen projektiven Gruppe $K_{z}$ und bilden das vollständige System der irreduziblen Relationen dieser Art. ${ }^{2}$ ) Ihre Anzahl ist 5 .

Die Relationen (3) und (4) zusammen gehören in demselben Sinne zu derjenigen Gruppe $K_{6}$ von Kollineationen, welche die Gerade $l$ in Ruhe lassen. Ihre Anzahl ist 11.

Ebenso gehören die Relationen (3) und (5) zusammen zu derjenigen Gruppe $K_{6}^{*}$ von Kollineationen, welche den Punkt $\lambda$ in Ruhe lassen. Ihre Anzahl ist gleichfalls 11.

Endlich gehören die sämtlichen Relationen (3)-(6) zu der von uns betrachteten Gruppe $K_{5}$ von Kollineationen. Ihre Anzahl ist 20.

Zu jeder der vorstehend aufgeführten Relationen kommt in der obigen Tabelle auch die dualistisch entsprechende vor, d. h. diejenige, welche aus ihr entsteht, wenn man die Punkte $x_{1}, x_{2}, x_{3}, x_{4} ; x, y, z ; \lambda$ gleichzeitig beztiglich mit den Ebenen $u_{1}, u_{2}, u_{3}, u_{4} ; u, v, w ; l$ vertauscht. Da das gleiche auch schon $\nabla 0 n$ den Typen (2) galt, so können wir sagen:

In der Mannigfaltigkeit der ganzen Invarianten der Gruppe $K_{5}$ herrscht vollkommene Dualität.

Das war übrigens vorauszusehen.

3. Wir wählen jetzt insbesondere als zu betrachtende Ebene die Minimalebene

$$
y+i z=0, \quad\left\{i^{2}=-1\right\}
$$

1) Das Auftreten der Relationen (6) ist eine Folge der Bedingung (1) zwischen den Koeffizienten der linearen Formen $(l x)$ und $(u \lambda)$. Solche Abhängig* keiten werden in der gewöhnlichen Theorie der ternären Formen $\mathrm{n} i \mathrm{ch}$ t vorausgesetzt. (Vgl. auch Study, BJ., S. 653.)

2) E. Study, Methoden zur Theorie der ternären Formen, Leipzig 1889, II, § 6 (\$. $74-83$ ). 
wenn $x, y, z$ die rechtwinkligen Cartesischen Koordinaten eines Punktes im komplexen Euklidischen Raume sind. Die homogenen Koordinaten $x_{1}: x_{2}: x_{3}$ eines Punktes $x$ im komplexen ternären Gebiet der Minimalebene (7) führen wir ein durch:

$$
\left.x=i \frac{x_{2}}{x_{1}}, y=\frac{x_{3}}{x_{1}}, z=i \frac{x_{3}}{x_{1}}{ }^{1}\right)
$$

und nennen $u_{1}: u_{2}: u_{3}$ die zugehörigen Linienkoordinaten, so daß

$$
(u x)=0
$$

die Bedingung der vereinigten Lage eines Punktes $x$ und einer geraden Linie $u$ darstellt.

Als Gerade $l$ oder

$$
(l x)=0
$$

führen wir die uneigentliche Gerade ${ }^{2}$ ) der Ebene (7) ein, also diejenige von den Koordinaten:

$$
l_{1}: l_{2}: l_{3}=1: 0: 0
$$

und als Punkt $\lambda$ oder

$$
(u \lambda)=0
$$

den absoluten Punkt der Minimalebene. Seine Koordinaten sind:

$$
\lambda_{1}: \lambda_{2}: \lambda_{3}=0: 0: 1 \text {. }
$$

Wir nennen ferner uneigentliche Punkte alle Punkte der absoluten Geraden, also alle Punkte von den Koordinaten $\left(0: x_{2}: x_{3}\right)$ einschlie $b 1$ ich des absoluten Punktes; und M in imalgeraden alle Geraden durch den absoluten Punkt, also alle Geraden von den Koordinaten $\left(u_{1}: u_{2}: 0\right)$, einschließlich der uneigentlichen Geraden. Ein Punkt $x$, für den $(l x) \equiv x_{1} \neq 0$ ist, heife ein eigentlicher Punkt, und eine Gerade $u$, für die $(u \lambda) \equiv u_{3} \neq 0$ ist, eine Euklidische Gerade. Werte:

Die Invarianten $(l u v)$ und $(x y \lambda)$ haben jetzt die folgenden

$$
(l u v)=u_{2} v_{3}-u_{3} v_{2}, \quad(x y \lambda)=x_{1} y_{2}-x_{2} y_{1} .
$$

Die erste bedeutet, gleich Null gesetzt, geometrisch, daß die beiden Geraden $u, v$ parallel sind (sich in einem uneigentlichen Punkte schneiden), die $z w e i t e$, daß die beiden Punkte $x, y$ auf einer Minimalgeraden liegen.

Unter den gemachten Voraussetzungen besteht die Gruppe $K_{6}^{*}$ (Nr. 1) aus denjenigen automorphen Kollineationen der Minimalebene,

1) H. Beck, ME., S. $14 \mathrm{f}$.

2) Uueigentliche Gerade der Minimalebene nennen wir ihre Schnittgerade mit der uneigentlichen Ebene, a bs ol a ten Punkt ibren Berührungspunkt mit dem absoluten Kegelschnitt des Eaklidischen Raumes. 
welche ihren absoluten Punkt in Ruhe lassen, die Gruppe $K_{6}$ aus den automorphen Affinitäten und die Gruppe $K_{5}$ aus den auto-. morphen Ähnlichkeiten der Minimalebene. Die Entwicklungen der beiden vorhergehenden Nummern enthalten somit auch die Theorie der ganzen Ähnlichireitsinvarianten einer Minimalebene. ${ }^{1}$ )

4. Es handelt sich nunmehr darum, auch die einfachsten absoluten Ähnlichkeits- und Bewegungsinvarianten der Minimalebene (7) aufzustellen; d. h. jene Quotienten ganzer Ähnlichkeitsinvarianten im System von zwei und drei Elementargebilden (Punkten und Geraden), die homogene Funktionen nullten Grades der Koordinaten dieser Elementargebilde sind und ungeändert bleiben, wenn man sie den Ähnlichkeiten oder Bewegungen einer der gleich aufzuzählenden Untergruppen der automorphen Ähnlichkeiten unterwirft.

$\mathrm{Zu}$ diesem Zwecke ist es notwendig, die Gleichungen der automorphen $\ddot{A} h n l i c h k e i t e n$ der Minimalebene wirklich anzuschreiben. Sie lauten ${ }^{2}$ ) in den Punktkoordinaten $x_{i}$ :

$$
\begin{aligned}
& x_{1}^{\prime}=b_{11} x_{1} \\
& x_{2}^{\prime}=b_{21} x_{1}+b_{22} x_{2} \\
& x_{3}^{\prime}=b_{31} x_{1}+b_{32} x_{2}+b_{33} x_{3}
\end{aligned}
$$

und in den Linienkoordinaten $u_{i}$ :

$$
\left.\begin{array}{lrr}
u_{1}^{\prime}=b_{22} b_{33} u_{1}-b_{33} b_{21} u_{2}+\left(b_{21} b_{32}-b_{31} b_{22}\right) u_{3} \\
u_{2}^{\prime}= & b_{33} b_{11} u_{2} & -b_{11} b_{32} u_{3} \\
u_{3}^{\prime}= & b_{11} b_{22} u_{3}
\end{array}\right\} .
$$

Die oben erwähnten Untergruppen der automorphen Ähnlichkeiten (13) sind die folgenden:

1. Die viergliedrige Grenzgruppe: ${ }^{3}$ )

$$
b_{33}=b_{22} \text {. }
$$

2. Die viergliedrige Gruppe der automorphen Bewegungen der Minimalebene

$$
b_{22}=b_{11} \text {. }
$$

1) Wir bemerken hier beiläufig, daß die Theorie der ganzen Ähulichkeitsinvarianten in einer Minimalebene, auch abgesehen von der in Nr. 2 erwäbnten Dualität, prinzipiell einfacher ist als die Theorie der ganzen Ähnlichkeitsinvarianten in einer Euklidischen Ebene. In dieser gibt es 8 Typen von elementaren Invarianten, zwischen denen 32 irreduzible Identitäten beștehen (E. Study, BJ., S. 652 and 657); in der Minimalebene gibt es nur 7 Typen, die durch 20 irreduzible Identitäten verbunden sind. Der Kern der Sache war natürlich vorauszusehen.

2) H. Beck; ME., S. 16, Formeln (8) und (9). Die Formeln (9) sind in der zweiten Zeile a. a. O. mit einem Druckfehler behaftet.

3) H. B eck, ME., S. $24 \mathrm{ff}$. 
3. Die viergliedrige Gruppe der $\ddot{A} h n l i c h k e i t s t r a n s-$ formationen, für die

$$
b_{33}=b_{11}
$$

ist ("Dritte Gruppe").

4. Der gemeinsame dreigliedrige Durchschnitt dieser drei Gruppen, die Bewegungen der Grenzgruppe: ${ }^{1}$ )

$$
b_{33}=b_{22}=b_{11} \text {. }
$$

Außer diesen Untergruppen der automorphen Ähnlichkeitstransformationen verdienen noch die gleichfalls in diesen Transformationen enthaltenen Scharen (Nicht-Gruppen!) der a u tomorphen Umlegungen der Minimalebene hervorgehoben $\mathrm{zu}$ werden. Sie sind definiert durch:

$$
b_{22}=-b_{11} .
$$

Insbesondere sind die Umlegungen der "dritten Gruppe" definiert durch:

$$
-b_{33}=b_{22}=-b_{11}
$$

und die Umlegungen der Grenzgruppe durch:

$$
b_{33}=+b_{22}=-b_{11} \text {. }
$$

Das Produkt zweier automorpher Umlegungen der Minimalebene ist eine automorphe Bewegung; dasjenige zweier Umlegungen der dritten Gruppe oder zweier Umlegungen der Grenzgruppe eine Bewegung der Grenzgruppe.

Um an die Bildung der absoluten Ähnlichkeits- und Bewegungsinvarianten schreiten zu können, müssen wir schließlich noch angeben, mit welchen Faktoren unsere Typen (2) durch die Ähnlichkeitstransformationen (13) reproduziert werden. Man hat:

$$
\left.\begin{array}{rlrl}
\left(u^{\prime} x^{\prime}\right)=b_{11} b_{22} b_{33} .(u x) & \\
\left(x^{\prime} y^{\prime} z^{\prime}\right) & =b_{11} b_{22} b_{33} \cdot(x y z), & & \left(u^{\prime} v^{\prime} w^{\prime}\right)=b_{11}^{2} b_{22}^{2} b_{33}^{2} .(u v v), \\
\left(l x^{\prime}\right) & =b_{11}(l x), & & \left(l u^{\prime} v^{\prime}\right)=b_{11}^{2} b_{22} b_{33} .(l u v), \\
\left(x^{\prime} y^{\prime} \lambda\right) & =b_{11} b_{22}(x y \lambda), & & \left(u^{\prime} \lambda\right)=b_{11} b_{22}(u \lambda) .
\end{array}\right\}
$$

5. Wir zählen nunmehr, zunächst für die Systeme von zwei beliebigen Elementargebilden, die einfachsten Größen auf, welche zum mindesten für die engste unserer Gruppen (die Bewegungen der Grenzgruppe) den Charakter absoluter Invarianten besitzen. Wir geben dabei bei jeder Invariante an, für welche Untergruppe der automorphen Ähnlichkeiten sie eine absolute Invariante ist und mit welchem Faktor sie durch die allgemeinste

1) Die Gruppen 1., 2. and 4. werden bei H. Beck, ME, ausdrücklich besprochen, die Gruppe 3. nicht. - Die Definitionsgleichung (15*) der automorphen Umlegungen findet sich ME., S. 16. 
antomorphe Ähnlichkeit (13) der Minimalebene multipliziert wird; endlich auch, welchen besonderen Systemen von Elementargebilden besondere Werte der Invariante zukommen.

1. Zwei Punkte $p, q$.

Der Abstand $\overline{p q}$ des Punktes $p$ rom Punkte $q$ :

$$
\overline{p q}=-\overline{q p}=i \frac{(p q \lambda)}{(l p)(l q)} \cdot{ }^{1}
$$

Absolute Invariante gegenüber allen automorphen Bewe gungen der Minimalebene:

$$
\overline{p^{\prime} q^{\prime}}=\frac{b_{22}}{b_{11}} \cdot \overline{p q}
$$

$\overline{p q}$ ist Null, wenn $p$ und $q$ eigentliche Punkte einer und derselben Minimalgeraden sind; unendlich groh, wenn einer (und nur einer) der beiden Punkte ein nicht-absoluter uneigentlicher Punkt ist; unbestimmt, wenn einer von ihnen der absolute Punkt ist oder wenn beide Punkte uneigentliche sind.

2. Ein Punkt $p$ und eine Gerade $a$.

Die Öffnung $(a, p)$ zwischen der Geraden $a$ und dem Punkte $p$ :

$$
(a, p)=(p, a)=i \frac{(a p)}{(l p)(a \lambda)} .
$$

Absolute Invariante gegenüber allen $\ddot{A} h n l i c h k e i t e n$ der „dritten Gruppe":

$$
\left(a^{\prime}, p^{\prime}\right)=\frac{b_{33}}{b_{11}} \cdot(a, p)
$$

$(a, p)$ ist Null, wenn $p$ ein eigentlicher Punlkt und $a$ eine Euklidische Gerade ist und beide auberdem vereinigt liegen; unendlich grof, wenn $p$ ein uneigentlicher Punkt und $a$ eine ibn nicht enthaltende Gerade ist, oder $a$ eine Minimalgerade und $p$ ein nicht auf ihr liegender Punkt; unbestimmt, wenn (mindestens) $p$ ein uneigentlicher Punkt oder $a$ eine Minimalgerade ist und $a$ und $p$ vereinigt liegen.

3. Zwei Gerade $a, b$.

Die Sperrung $\overparen{a b}$ der Geraden $a$ und $b$ :

$$
\overparen{a b}=-\overparen{b a}=-\frac{(l a b)}{(a \lambda) \bar{b} \lambda)}
$$

I) Schon aus dieser Formel geht hervor, daß das von uns "Gerade" genannte Elementargebilde, vom Standpunkte der Euklidischen Raumgeometrie eine in bestimmter Weise orientierte Gerade, ein Speer ist. [Man würde überall die entgegengesetzten Speere erhalten, wenn man $(u \lambda)=-u_{3}$ gesetzt hätte.] 
Absolute Invariante gegenüber allen Ähnlichkeiten der Grenzgruppe:

$$
\widehat{a^{\prime} b^{\prime}}=\frac{b_{33}}{b_{22}} \widehat{a b}
$$

$\overparen{a b}$ ist Null, wenn $a$ und $b$ parallele Euklidische Gerade sind; unendlich groß, wenn eine (und nur eine) der beiden Geraden eine eigentliche Minimalgerade ist; unbestimmt, wenn eine von ihnen die uneigentliche Gerade ist, oder wenn beide Geraden Minimalgeraden sind. ${ }^{1}$ )

6. Aus den Entwicklungen der letzten Nummer geht hervor, daß in der Minimalebene die Bew eg ungen der Grenzgruppe die allgemeinste Untergruppe der automorphen Ähnlichkeitstransformationen sind, hinsichtlich deren bereits zwei beliebige Elementargebilde eine absolute Invariante besitzen ${ }^{2}$ ): im Gegensatze zur Euklidisehen Ebene, wo diese Eigensehaft sämtlicheul automorphen Bewegungen zukommt. Eine Elementargeometrie, die in ihrer Struktur der Elementargeometrie der Euklidischen Ebene (d. h. der Geometrie ihrer Bewegungsgruppe ${ }^{3}$ entspricht, läbt sich somit inder Minimalebene nur dann aufbauen, wenn man ihr als Fundamentalgruppe die (besonderen) Bewegungen der Grenzgruppe zu Grunde legt, nicht aber die automorphen Bewegungen schlechthin.

In dieser "Elementargeometrie in der Minimalebene" (Geometrie der Bewegungen der Grenzgruppe) herrseht nun, wie die Formeln der vorhergehenden Nummer zeigen, eine vollk ommene metrische Dualität: Es entsprechen einander als dualistische Gegenstücke nicht nur, wie es bereits in der Geometrie der automorphen Ähnlichkeiten der Minimalebene der Fall war (rgl. Nr. 2):

ein eigentlicher Punkt

ein uneigentlicher Pnnkt

die uneigentliche Gerade eine Euklidische Gerade

eine Minimalgerade

der absolute Punkt,

1) Die hier und in Nr. 7 angeführten absoluten Invarianten treten zu m Teil bereits bei Herrn Beck auf (die Sperrung ME., S. $16 \mathrm{f}$, die Fassung S. 20 , die Abweichung S. 18 f.); die Öffung und die Invariante $\Phi$ eines Dreiseits werden dagegen in der vorliegenden Arbeit neu eingeführt.

2) Gegenüber einer beliebige n automorphen Bewegung der Minimalebene hat vur die Figur zweier (eigentlicher) Purkte, nicht aber die Kigur eines (eigentlichen) Punktes und einer (Euklidischen) Geraden oder diejenige zweier (Euklidischer) Geraden, eine absolute Invariante.

$\left.{ }^{3}\right)$ Wir fassen den Begrift der Elementargeometrie der Euklidischen Ebene bier absichtlich etwas enger, als das in der Regel - nach dem Vorgange von F. Klein (Erlanger Programm) - geschieht, wo man unter Elementargeometrie die Geometrie der Hauptgrup pe versteht. 
sondern auch:

der Abstand zweier Punkte f die Öffnung zwischen einem Punkte und einer Geraden die Sperrung zweier Geraden f die Öffnung zwischen einer GGeraden und einem Punkte.

Aus jeder Größe auf der linken Seite der vorstehenden Tabellen geht nämlich durch die Korrelation:

$$
u_{1}=x_{3}, \quad u_{2}=i x_{2}, \quad u_{3}=x_{1},
$$

die entsprechende Größe auf der rechten Seite hervor. Die Korrelation (22), gleichzeitig angewandt auf einen Punkt $x$ und den aus ihm durch eine Bewegung der Grenzgruppe hervorgehenden Punkt $x^{\prime}$, gibt eine Gerade $u_{*}$ und die aus ihr durch eine (im allgemeinen von der ersten verschiedene) Bewegung der Grenzgruppe hervorgehende Gerade $u t^{\prime}$. -

Gegenüber den a utomorphen Umlegungen der Minimalebene und den in ihnen enthaltenen Scharen von Transformationen verhalten sich die in der letzten Nummer aufgezählten Invarianten folgendermaßen:

Eine beliebige a utomorphe Umlegung führt den Abstand in seinen entgegengesetzten Wert über, während sie Öffnung und Sperrung mit einem (im allgemeinen von $\$ 1$ verschiedenen) Faktor multipliziert.

Die Umlegungen der „dritten Gruppe" lassen die Öffnung unverändert und multiplizieren die Sperrung mit -1 ; die Umlegungen der Grenzgruppe lassen umgekehrt die Sperrung unverăndert und multiplizieren die Öffnung mit -1 .

Es gibtkeineautomorphe Umlegung, welebe $A$ bstand, Öfnung, Sperrung sämtlich mit - 1 multipliziert.

7. Wir betrachten jetzt noch die Figur dreier Punkte $p q r$ oder dreierGeraden $a b c\{\hat{q r}=a, \hat{r} p=b, \widehat{p q}=c\}$ - Dreieck oder Dreiseit im weiteren Sinne ${ }^{1}$ - auf ihre einfachsten absoluten Invarianten hin.

Wir zählen zunächst nochmals die bereits in Nr. 5 definierten Invarianten auf, indem wir dabei Punkt- und Linienkoordinaten gleichmäßig berïcksichtigen :

1. Die Längen der Seiten $\overline{q r}, \overline{r p}, \overline{p q}$ :

$$
\overline{q r}=i \frac{(q r \lambda)}{(l q)(l r)}=i \frac{(a b c)(a \lambda)}{(l c a)(l a b)} \quad \text { usw. }
$$

1) Wir werden später, aus einem Grunde, der aus dieser Nummer unmittelbar ersichtlich ist (Versehwinden, Unendlich- oder Unbestimmtwerden wenigstens einer der aufgezahlten Invarianten) die Begriffe Dreieck und Dreis i t wesentlich enger begrenzen ( $\mathrm{Nr} .9$ ). 
2. Die Öffnungen $(a, p),(b, q),(c, r)$ zwischen einer Seite und der gegenüberliegenden Ecke:

$$
(a, p)=i \frac{(a p)}{(l p)(a \lambda)}=i \frac{(p q r)}{(l p)(q r \lambda)}=i \frac{(a b c)}{(a \lambda)(l b c)} \text { usw. }
$$

3. Die "Sperrungen in den Ecken" $\overparen{b c}, \overparen{c a}, \overparen{a b}$ :

$$
\widehat{b c}=-\frac{(p q r)(l p)}{(r p \lambda)(p q \lambda)}=-\frac{(l b c)}{(b \lambda)(c \lambda)} \quad \text { usw. }
$$

Die nicht angeschriebenen Größen gehen aus den angeschriebenen durch gleichzeitige zyklische Vertauschung der Buchstaben $p, q, r$ untereinander und der Buchstaben $a, b, c$ untereinander hervor.

$\mathrm{Zu}$ diesen Invarianten kommen noch einige neue:

4. Die Fassung $F$ des Dreiecks $p q r$ :

$$
F=-\frac{1}{2} \frac{(p q r)}{(l p)(l q)(l r)}=-\frac{1}{2} \frac{(a b c)^{2}}{(l b c)(l c a)(l a b)} \text {. }
$$

Absolute Invariante gegenüber allen Bewegungen der Grenzgruppe:

$$
F^{\prime}=\frac{b_{22} \cdot b_{33}}{b_{11}^{2}} \cdot F
$$

$F$ ist Null, wenn die drei Punkte $p, q, r$ eigentliche Punkte sind und in einer Geraden liegen; unendlich groh, wenn mindestens einer der drei Punkte uneigentlich ist, ohne daß die drei Punkte einer Geraden angehören; unbestimmt, wenn die drei Punkte in einer Geraden liegen und mindestens einer von ihnen uneigentlich ist.

5. Das dualistische Gegenstick (Nr. 6) der Fassung eines Dreiecks, die Invariante $\Phi$ des Dreiseits $a b c$ :

$$
\Phi=-\frac{i}{2} \frac{(a b c)}{(a \lambda)(b \lambda)(c \lambda)}=-\frac{i}{2} \frac{(p q r)^{2}}{(q r \lambda)(r p \lambda)(p q \lambda)} .
$$

Absolute Invariante gegenüber allen Bewegungen der Grenzgrupe:

$$
\Phi^{\prime}=\frac{b_{33}^{2}}{b_{11} \cdot b_{32}} \cdot \Phi
$$

$\Phi$ ist Null, wenn die drei Geraden $a, b, c$ Euklidische Gerade sind und durch einen Punkt gehen; unendlich groß, wenn mindestens eine der drei Geraden eine Minimalgerade ist, ohne daß die drei Geraden einem Büschel angehören; unbestimmt, wenn die drei 
Geraden durch einen Punkt gehen und mindestens eine von ihnen eine Minimalgerade ist.

6. Die Abweichung $A$ des parabolischen Umkreises ${ }^{1}$ ) des Dreiecks $p q r$ :

$$
A=-2 i \frac{(p q r)(l p)(l q)(l r)}{(q r \lambda)(r p \lambda)(p q \lambda)}=-2 i \frac{(l b c)(l c a)(l a b)}{(a b c)(a \lambda)(b \lambda)(c \lambda)}
$$

Absolute Invariante gegenüber allen Bewegungen der Grenzgruppe:

$$
A^{\prime}=\frac{b_{33} b_{11}}{b_{22}^{2}} \cdot A
$$

$A$ ist Null, wenn die Punkte $p, q, r$ sämtlich eigentliche Punkte einer Euklidischen Geraden sind oder wenn einer (und nur einer) der drei Punkte ein nicht-absoluter uneigentlicher ist, während die übrigen eigentliche Punkte einer Euklidischen Geraden sind; unendlich grol, wenn eines (und nur eines) der drei Paare $q r, r p, p q$ eigentliche Punkte einer Minimalgeraden sind; unbestimmt, wenn $p, q, r$ sämtlich eigentliche Punkte einer Minimalgeraden sind oder wenn mindestens zwei der drei Punkte nicht-absolute uneigentliche Punkte sind oder endlich, wenn einer der drei Punkte der absolute Punkt ist. ${ }^{2}$ )

7. Die Abweichung A des parabolischen Inkreises des Dreiseits $a b c$ :

$$
\mathrm{A}=-\frac{\dot{b}}{2} \frac{(l b c)(l c a)(l a b)}{(a b c)(a \lambda)(b \lambda)(c \lambda)}=-\frac{i}{2} \frac{(p q r)(l p)(l q)(l r)}{(q r \lambda)(r p \lambda)(p q \lambda)}
$$

1. Absolute Invariante gegenüber allen Bewegungen der Grenzgruppe:

$$
A^{\prime}=\frac{b_{33} b_{11}}{b_{22}^{2}} \cdot A \text {. }
$$

1) Unter den (irreduziblen) parabolischen oder singulären Kreisen (Study) versteht man diejenigen irreduziblen Kurven zweiten Grades in der Minimalebene, welche die uneigentliche Gerade im absoluten Punkte berïhren. Alle absoluten Invarianten eines parabolischen Kreises gegenüber den Bewegungen der Grenzgruppe sind Funktionen einer einzigen, der $\mathrm{A}$ b w e ic b u g des parabolischen Kreises (H, B eck, ME., S. 18 f., wo die Abweichung für beliebige analytische krumme Linien in der Minimalebene definiert wird). - Vgl. auch Nr. 8.

2) In allen diesen Fällen entartet entweder der (als Ordnungskurve aufzufassende) parabolische Umkreis des Dreiecks, oder er ist nicht völlig bestimmt. Er entartet in eine Euklidische Gerade und die uneigentliche Gerade, wenn $A$ Null ist; in zwei eigentliche Minimalgerade, wenn $A$ unendlich groß ist. Wenn endlich $A$ unbestimmt ist, so entartet entweder der Umkreis in eine eigentliche Minimalgerade und die uneigentliche Gerade oder, im besonderen Falle, in die doppelt-zählende uneigentliche Gerade; oder das Dreieck besitzt mehr als einen Umkreis. Wir überlassen es dem Leser, sich bei unbestimmtem $A$ in jedem einzelnen Falle zu überzeugen, was eintritt. 
A ist Null, wenn eines (und nur eines) der drei Paare $b c, c a, a b$ parallele Euklidische Gerade sind; unendlich groß, wenn die Geraden $a, b, c$ sämtlich Euklidische Gerade durch einen eigentlichen Punkt sind oder wenn eine (und nur eine) der drei Geraden eine eigentliche Minimalgerade ist, während die anderen nicht-parallele Euklidische Gerade sind; unbestimmt, wenn alle drei Geraden parallele Euklidische Gerade sind oder wenn mindestens zwei der drei Geraden eigentliche Minimalgerade sind oder endlich, wenn eine der drei Geraden die uneigentliche Gerade ist. ${ }^{1}$ )

Dualistische Gegenstücke (Nr.6) sind $A$ und $\frac{1}{\mathrm{~A}}$.

Das Verhalten der in dieser Nummer neu eingeführten Invarianten $F, \Phi, A$, A gegenüber den a u tomorphen Umlegun$\mathrm{g}$ en der Minimalebene ist folgendes:

Eine beliebige automorphe Umlegung multipliziert sie sämtlich mit einem (im allgemeinen von -1 verschiedenen) Faktor.

Eine Umlegung der "dritten Gruppe" multipliziert $F$ und $\Phi$ mit -1 und läßt $A$ und A ungeändert. Also (vgl. Nr. 8): Die Abweichung eines beliebigen parabolischen Kreises ist eine absolute Invariante gegenüber allen Umlegungen der dritten Gruppe.

Eine Umlegung der Grenzgr up pe multipliziert $\Phi, A, \mathrm{~A}$ mit -1. Die Fassung $F$ eines Dreiecks ist eine absolute Invariante auch gegenüber allen Umlegungen der Grenzgruppe.

Endlich ist die Invariante $\Phi$ eines Dreiseits eine absolute Invariante gegenüber denjenigen beiden getrenten Scharen von Umlegungen, für welche

$$
\pm i b_{33}=b_{22}=-b_{11}
$$

ist. Die erste dieser Scharen (oberes Zeichen) multipliziert $F$ mit $-i, A$ und $\mathrm{A}$ mit $i$; die zweite umgekehrt $F$ mit $i, A$ and $A$ mit $-i$.

8. Wir betrachten jetzt diejenigen geometrischen $\ddot{O}$ rter von Punkten und Geraden, für welche die in den Nummern 5 und 7 eingeführten absoluten Invarianten konstante, endliche, von Null verschiedene Werte besitzen. Dabei stellen wir dualistische Gegenstücke (Nr. 6) einander gegeniiber.

1) In allen diesen Fällen entartet entweder der (als Klassenkurvo aufza. fassende) parabolische Inkreis des Dreiseits, oder er ist nicht völlig bestimmt. Er entartet in zwei nicht-absolute uneigentliche Punkte, wenn A Null ist; in einen eigentlichen Punkt und den absoluten Punkt, wenn A unendlich gro B ist. Wenn endlich A unbestimmt ist, so entartet entweder der Inkrois in einen nicht-absoluten und den absoluten uneigentlichen Punkt oder, im besonderen Falle, in den doppeltzählenden absolaten Punkt; oder das Dreiseit besitzt mehr als einen Inkreis. 
1a) Der Ort aller Punkte $x$, welche von einem eigentlichen Punkte $p$ konstanten Abstand $c^{1}$ ) haben, ist die Minimalgerade:

$$
(x p \lambda)+i c(l p)(l x)=0 \text {. }
$$

2a) Der Ort aller Punkte $x$, welche mit einer Euklidischen Geraden $a$ konstante Öffnung $c$ haben, ist die Parallele

$$
(a x)+i c(l x)(a \lambda)=0
$$

zu $a$.

3a) Der Ort aller Punkte $x$, welche mit zwei gegebenen eigentlichen Punkten $p, q$ ein Dreieck von konstanter Fassung $F$ bilden, ist die Parallele:

$$
(p q x)+2 F(l p)(l q)(l x)=0
$$

zur Geraden $p q$.

4a) Der Ort aller Punkte $x$, welche mit zwei gegebenen Punkten $p, q$ einer Euklidischen Geraden ein Dreiseit von konstanter Invariante $\Phi$ bestimmen, ist der irreduzible Kegelschnitt:

$$
\begin{gathered}
(p q x)^{2}+ \\
+2 i \Phi(p q \lambda)(x p \lambda)(x q \lambda)=0
\end{gathered}
$$

der die Gerade $\widehat{p} q$ zur Hauptachse (Polare des absoluten Punktes) und $p, q$ zu Pseudobrennpunkten (nicht-absoluten Berührungspunkten der Tangenten durch den absoluten Punkt) hat. ${ }^{2}$ )

Sind $p, q$ beide eigentliche Punkte, so ist der Kegelschnitt ein Mittelpunktskegelschnitt 1b) Der Ort aller Geraden $u$, welche gegen eine Euklidische Gerade a konstante Sperrung $c$ haben, ist der uneigentliche Punkt:

$(l u a)+c(a \lambda)(u \lambda)=0$.

2b) Der Ort aller Geraden $u$, welche mit einem eigentlichen Punkt $p$ konstante Öffnung $c$ haben, ist der eigentliche Punkt

$$
(u p)+i c(l p)(u \lambda)=0(2 \measuredangle b)
$$

auf der Minimalgeraden durch $p$.

3b) Der Ort aller Geraden $u$, welche mit zwei gegebenen $\mathrm{Eu}$ klidischen Geraden $a, b$ ein Dreiseit von konstanter Invariante () bilden, ist der Punkt:

$(a b u)-2 i \Phi(a \lambda)(b \lambda)(a \lambda) \stackrel{(29 b)}{=0}$ $(a b u)-2 i \Phi(a \lambda)(b \lambda)(u \lambda)=0$ auf der Minimalgeraden des Punktes $p q$.

4b) Der Ort aller Geraden $u$, welche mit zwei gegebenen nichtparallelen Geraden $a, b$ ein Dreieck von konstanter Fassung $F$ bestimmen, ist der irreduzible Kegelschnitt :

$$
(a b u)^{2}-
$$

- $2 F(l a b)(l a u)(l b u)=0$,

der den Punkt $a b$ zum Mittelpunkt (Pol der uneigentlichen Geraden) und $a, b$ zu $\mathrm{Asym}$ ptoten (eigentlichen Tangenten in den Schnittpunkten mit der uneigentlichen Geraden) hat.

Sind $a, b$ beide Euklidische Gerade, so ist der Kegelschnitt ein Mittelpunktskegelschnitt

1) Die in dieser Nummer auftretenden Buchstaben $c, F, \Phi, A, \mathrm{~A}$ bezeichnen durchwegs endliche, von $\mathrm{Null}$ verschiedene Konstante.

2) Die Theorie der Kegelschnitte in der Minimalebene ist vom Verfasser in einer besonderen, noch nicht veröffentlichten Abhandlung ("Über die. Kegelschnitte in einer Minimalebene") ausfïhrlich behandelt worden. 
erster Art; ${ }^{1}$ ist einer von beiden ein uneigentlicher, nichtabsoluter Punkt, so ist er eine Parabel allgemeiner A r t. ${ }^{1}$ ) erster Art; ${ }^{1}$ ) ist einer ron beiden eine eigentliche Minimalgerade, so ist er ein Mittelpunktskegelschnitt zweiter Art. $\left.{ }^{1}\right)$

Als Kurven konstanter Abweichung $A(25)$ oder A (26) ergeben sich endlich die (irreduziblen) parabolischen Kreise:

Die Gleichung des parabolisehen Kreises von der (konstanten) Abweichung $A$ durch die Punkte $p, q$ ist:

$$
\begin{aligned}
& 2(l p)(l q)(l x)(p q x)+\quad(31 a) \\
& +A i(p q \lambda)(x p \lambda) \cdot(x q \lambda)=0 .
\end{aligned}
$$

Die Gleichung des parabolischen Umkreises eines Dreiecks $p q r$ kann, wenn er irreduzibel ist, in der Form $(l p)(q r \lambda)(p q x)(x r \lambda)-(32 a)$ $-(l r)(p q \lambda)(q r x)(x p \lambda)=0$

oder in den beiden hieraus durch zyklische Vertauschnng der Buchstaben $p, q, r$ hervorgehenden Formen geschrieben werden.
Die Gleichung des parabolischen Kreises von der (konstanten) Abweichung A und den Tangenten $a, b$ ist:

$$
\begin{aligned}
& 2 A i(a \lambda)(b \lambda)(u b)(a b u)+ \\
& +(l a b)(l a u)(l b u)=0 .
\end{aligned}
$$

Die Gleichung des parabolischen Inkreises eines Dreiseits $a b c$ kann, wenn er irreduzibel ist, in der Form $(a \lambda)(l b c)(a b u)(l c u)-$ $-(c \lambda)(l a b)(b c u)(l a u)=0$

oder in den beiden hieraus durch zyklische Vertauschung der Buchstaben $a, b, c$ hervorgehenden Formen geschrieben werden.

Auch die parabolisehen Kreise ergeben sich auf dem in dieser Nummer eingeschlagenen Wege als geometrische Örter von Punkten und Geraden:

Der Ort aller Punkte $x$, deren Verbindungslinien mit zwei gegebenen eigentlichen Punkten $p, q$ einer Euklidischen Geraden konstante Sperrung $c$ besitzen, ist der irreduzible parabolische Kreis:

$$
\begin{gathered}
(l x)(p q x)+ \\
+c(p x \lambda)(q x \lambda)=0
\end{gathered}
$$

durch die beiden gegebenen Punkte.
Der Ort aller Geraden $u$, deren Schnittpunkte mit zwei gegebenen nicht-parallelen Euklidischen Geraden $a, b$ konstanten Abstand $c$ besitzen, ist der irreduzible parabolische Kreis:

$$
\begin{gathered}
(u \lambda)(a b u)+ \\
+c i(l a u)(l b u)=0,
\end{gathered}
$$

der die beiden gegebenen Geraden berührt.

1) Außer den bereits definierten parabolischen Kreisen gibt es in der Minimalebene noch die folgenden gegenüber allen automorphen Ähnlichkeiten dieser Ebene invarianten Typen von irreduziblen Kegelschnitten:

a) Die Mittelpunktskgelschnitte erster Art, d, h. diejenigen Kegelschnitte, welche die uneigentliche Gerade in zwei (getrennten) Punkten schneiden und zwei (getrennte) Tangenten durch den absoluten Punkt besitzen. 
Die Sperrung $c$ ist dabei mit der Abweichung $A$ des parabolischen Kreises und dem Abstande $\overline{p q}$ der beiden gegebenen Punkte durch die Beziehung:

$$
c=\frac{1}{2} A \cdot \overline{p q}
$$

verbunden, wie ein Blick auf (31 a) zeigt.
Der Abstand $c$ ist dabei mit der Abweichung A des parabolischen Kreises und der Sperrung $\overparen{a b}$ der beiden gegebenen Geraden durch die Beziehung:

$$
c=\frac{1}{2 \mathrm{~A}} \cdot \overparen{a b}
$$

verbunden, wie ein Blick auf (31 b) zeigt.

9. Wir wenden schlieblich die Formeln $\left(19^{\prime}\right)-\left(21^{\prime}\right)$ und (23) - (26) in Nr.5 noch an, um Beziehungen $\mathrm{z}$ wischen den absoluten Invarianten eines Dreiecks oder Dreiseits (im engeren Sinne) aufzustellen, die ein Analogon zu bekannten planimetrischen und trigonometrischen Formeln der Euklidischen Ebene bilden. ${ }^{1}$ )

Wir verstehen dabei unter einem Dreieck die Gesamtheit dreier in eine bestimmte Reihenfolge gesetzter eigentlicher Punkte, die durch drei Euklidische Gerade verbunden werden können, 'und unter einem Dreiseit die Gesamtheit dreier in eine bestimmte Reihenfolge gesetzten Euklidischen Geraden, die sich in drei eigentlichen Punkten schneiden. Das Dreieck dreier Punkte ist mit dem Dreiseit ihrer Verbindungsgeraden identisch.

Seien $p, q, r$ die drei Punkte, $a, b, c$ die drei Geraden $\{a=\widehat{q r}, b=\widehat{r p}, c=\widehat{p q}\}$ des Dreiecks oder Dreiseits. Wir führen, um die Analogie mit der Euklidischen Ebene möglichst deutlich hervortreten zu lassen, die folgenden Bezeichnungen ein:

$$
\begin{gathered}
\overline{q r}=a, \overline{r p}=b, \overline{p q}=c, \\
\overparen{b c}=\alpha, \overparen{c a}=\beta, \overparen{a b}=\gamma, \\
(a, p)=h_{a}=h_{\alpha},(b, q)=h_{b}=h_{\beta},(c, r)=h_{c}=h_{\gamma}, \\
\frac{1}{A}=r, \frac{1}{\mathrm{~A}}=\rho .
\end{gathered}
$$

Dann folgen aus den oben aufgezählten Formeln, zum Teil mit Hilfe der Identitäten (3) - (6), die folgenden Relationen zwischen diesen Größen:

b) Die Mittelpunktskegelschnitte zweiter Art, d. h. diejenigen Kegelschnitte, welche durch den absoluten Punkt gehen, ohne die uneigentliche Gerade zu berühren.

c) Die Parabeln allgemeiner Art, d. h. diejenigen Kegelschnitte, welche die uneigentliche Gerade in einem nicht-absoluten Punkte berihhren.

1) Ein Teil dieser Beziehungen wurde schon von Herrn H. Beck aufgestellt (ME., S. 20 f.). 
(36)

$$
\begin{aligned}
& a: b: c=\alpha: \beta: \gamma, \\
& a+b+c=0, \quad \alpha+\beta+\gamma=0, \\
& 2 F=a \cdot h_{a}=\bar{b} \cdot h_{b}=c \cdot h_{c}=\quad 2 \Phi=\alpha \cdot h_{\alpha}=\beta \cdot h_{\beta}=\gamma \cdot h_{\gamma}= \\
& =-b c \cdot \alpha=-c a \cdot \beta=-a b \gamma=\quad=-\beta \gamma \cdot a=-\gamma \alpha \cdot b=-\alpha \beta \cdot c= \\
& =\frac{a b c}{2 r}=-4 r^{2} \cdot \alpha \beta_{\gamma}, \quad=\frac{\alpha \beta \gamma}{2} \cdot \rho=-\frac{4 a b c}{\rho^{2}}, \\
& b c=2 r h_{a}, c a=2 r h_{b}, a b=2 r h_{c}, \quad \beta \gamma=\frac{2 h_{\alpha}}{\rho}, \quad \gamma \alpha=\frac{2 h_{\beta}}{\rho}, \quad \alpha \beta=\frac{2 h_{\gamma}}{\rho}, \\
& p=4 r \\
& \text { usw. }
\end{aligned}
$$

Wir haben die elementare Geometrie in der Minimalebene (d. h. also die Geometrie der Bewegungen der Grenzgruppe) hier nur so weit entwickelt, als ihre Sätze sich als unmittelbare Folgerungen aus den Definitionen der absoluten Invarianten zweier und dreier Punkte und Geraden gegenüber dieser Gruppe ergaben. Es hat keine Schwierigkeit, diese Elementargeometrie weiter auszubauen, z. B. etwa die "Dreiecksgeometrie" in der Minimalebene zu studieren. Einen Versuch in anderer Richtung (Geometrie der Kegelschnitte in der Minimalebene) hat der Verfasser in der bereits zitierten, bisher unveröffentlichten Abhandlung gemacht. 